REVISTA PROYECCIONES N ${ }^{\circ}$ 12:57-70

Diciembre 1986 - ISSN 0716-0917

Jornada Matemáticas, Agosto 1986.

\title{
CONSTRUCCION DE UNA BASE DE SEGUNDO ORDEN USANDO EL METODO HOT PARA ELEMENTOS FINITOS TRIANGULARES CON UN LADO CURVO
}

\section{Dr. HECTOR ROJO JERALDO*}

\section{INTRODUCCION.}

En la construcción de funciones de base para elementos finitos curvos, el método isoparamétrico posee la ventaja de la simplicidad en la definición de la transformación $y$ en el hecho de que las funciones de base construidas usando la transformación isoparamétrica son polinomios. Esto hace que las típicas integrales que deben ser calculadas por algún método numérico no representen un gran problema. Sin embargo, el método isoparamétrico es esencialmente de primer orden. Los métodos directos tie nen la ventaja de ser capaces de aproximar la geometría de los lados curvos de los elementos finitos con mayor exactitud que el método isoparamétrico y las funciones de base construidas vía métodos directos, pueden ser de cualquier orden deseado [9]. La mayor desventaja de los métodos direc tos es que las funciones de base son usualmente funciones racionales, 10

* Académico Facuitad de Ciencias Básicas, Departamento de Matemáticas Universidad de Antofagasta. 
que hace que el proceso de integración sea mucho más complicado. Un méto do alternativo, el método de transformación de alto orden, el método H.O. T., permite que las funciones de base pueden ser construidas combinando las ventajas de la simplicidad del método isoparamétrico con la exactitud de los métodos directos, evitando el uso de funciones racionales [3].

\section{EI METODO HOT.}

El método de transformación de alto orden mapea el triángulo de tres lados rectos en el plano $p-q$ en un elemento de tres lados curvos en el plano s-t. Este método no está restringido a ninguna transformación en particular; sin embargo, la transformación isoparamétrica es la más sim ple de ser usada, la que luce como

$$
\begin{aligned}
& s=s(p, q) \\
& t=T(p, q)
\end{aligned}
$$

De acuerdo a Mc Leod [2], en general los grados de libertad en un polinomio de grado $n$ son menores que el número requerido de funciones de base, si uno quiere asegurar conformidad. Por ejemplo, en la construc ción de una base conforme para una aproximación de orden dos sobre un triángulu con un lado cónico y dos lados rectos, se necesitan ocho funcio nes de base y el número de grados de libertad es sólo de seis. Este hecho hace que el sistema de ecuaciones que demanda que las funciones de ba se expandan polinomios de grado 2 sea indeterminado.

El número de grados de libertad de un polinomio de grado $\mathrm{n}$ es

$$
M=(n+1)(n+2) / 2 \text {. }
$$

Si se tiene un elemento con $\mathrm{P}$ lados Y cada lado es una curva al ge braica de grado $m_{k}$, el número total de funciones de base para asegurar conformidad es 


$$
N=\sum_{k=1}^{P} N_{k}-P
$$

donde

$$
N_{k}=\frac{1}{2}\left[(n+1)(n+2)-u_{m_{k} n}\left(n-m_{k}+1\right)\left(n-m_{k}+2\right)\right]
$$

si podemos construir (N - M) funciones de base, entonces podemos resolver el sistema para las restantes.

Localizamos los nodos $\left\{\left(s_{i}, t_{i}\right)\right\}, i=1,2, \ldots, M$ en el elemento en el plano s-t de tal forma que estos nodos no se encuentren todos sobre una curva de grado $n$; entonces existe un único conjunto de polinomios $\left\{f_{i}(s, t)\right\}, i=1,2, \ldots, M$ de grado $n$ con la propiedad

$$
f_{i}\left(s_{j}, t_{j}\right)=\delta_{i j} ; \quad i, j=1,2, \ldots, M \text {. }
$$

Si $\left\{w_{i}(p, q)\right\}, i=M+1, M+2, \ldots, N$ son las funciones de base correspondien tes a los restantes nodos $\left\{\left(s_{i}, t_{j}\right)\right\}$, las funciones de base correspondientes a los nodos $1,2,3, \ldots, M$ son

$$
w_{i}(s, t, p, q,)=f_{i}(s, t)-\sum_{j=M+1}^{N} f_{i}\left(s_{j}, t_{j}\right) w_{j}(p, q)
$$

para $i=1,2,3, \ldots$.

Puésto que (1) expresa s y $t$ como polinomios en $p y q$, las funciones de base

$$
\left\{w_{i}(s, t, p, q)\right\}
$$

son polinomios en el plano $p-q$. La conformidad, orden $y$ más detalles de este método pueden ser encontrados en [1]. 
Como un ejemplo del método HOT, consideramos la construcción de una base de segundo orden para el elemento de tres lados mostrado en la figura 1, donde cada lado es un arco de parábola.

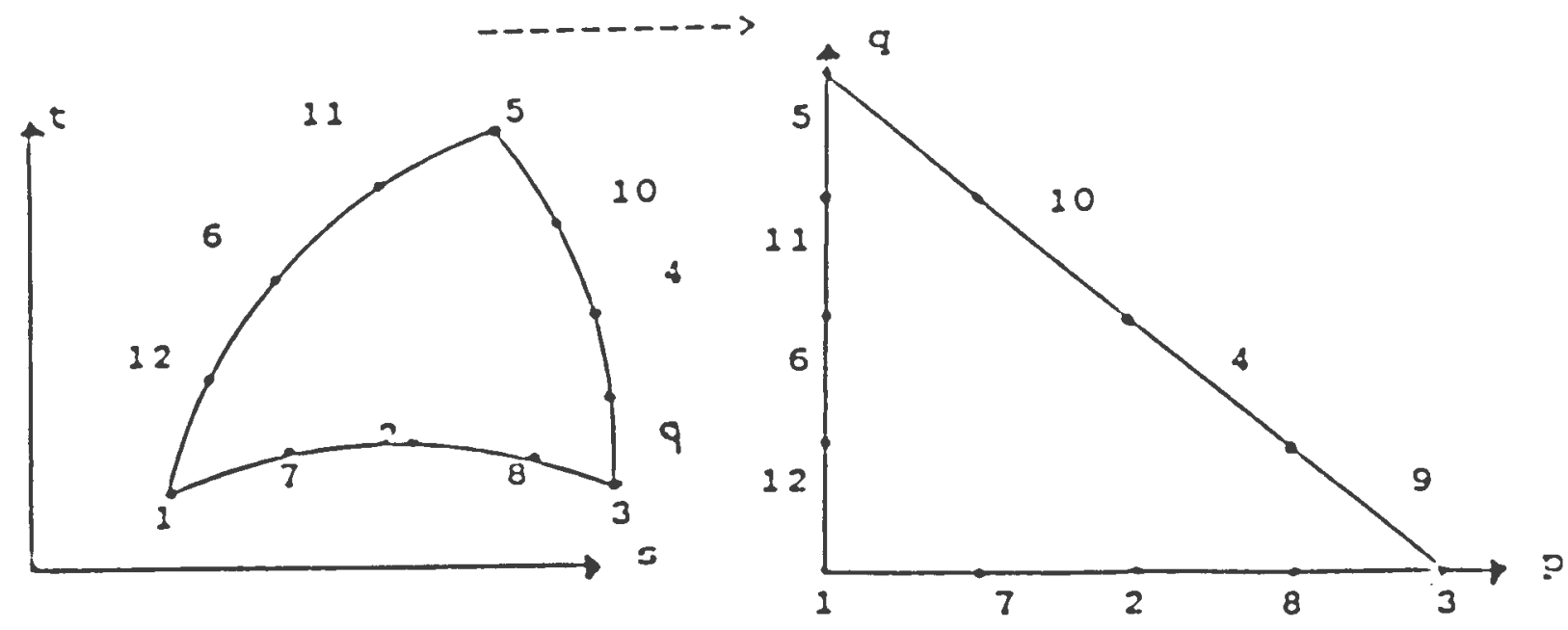

Figura 1: Posiciones nodales para una aproximación de segundo orden.

Debido a que cada lado del elemento en el plano s-t es un arco parabólico, se necesitan cinco nodos sobre cada lado, con un total de doce para asegurar conformidad. Los nodos son elegidos igualmente espaciados a lo largo de los lados del elemento. Se usa la transformación isopa ramétrica usual para mapear el elemento con tres lados rectos sobre el elemento curvo. La transformación es de la forma

$$
\begin{aligned}
& s=\sum_{i=1}^{6} s_{i} w_{i}^{*}(p, q)=s(p, q) \\
& t=\sum_{i=1}^{6} t_{i} w_{i}^{*}(p, q)=T(p, q)
\end{aligned}
$$


donde las funciones $w_{i}^{*}(p, q)$ son las funciones de base usuales dadas por

$$
\begin{aligned}
& w_{1}^{*}(p, q)=(1-p-q)(1-2 p-2 q) \\
& W_{2}^{*}(p, q)=4 p(1-p-q) \\
& W_{3}^{*}(p, q)=p(2 p-1) \\
& W_{4}^{*}(p, q)=4 p q \\
& W_{5}^{*}(p, q)=q(2 p-1) \\
& W_{6}^{*}(p, q)=4 q(1-p-q)
\end{aligned}
$$

$y$ así $s(p, q), T(p, q)$ son polinomios de grado dos en el plano $p-q$. Sea $(i ; j)_{k}$ la forma lineal en las variables $s, t$ con valor cero en los nodos $i, j y$ con valor 1 en el nodo $k$. Definimos las funciones $w_{i}(p, q)$

$i=M+1, M+2, \ldots, N$, donde $M=6$ y $N=12$ como sigue:

$$
\begin{aligned}
& w_{7}(p, q)=16 p(1-p-q)(2 ; 8)_{7} / 3 \\
& w_{8}(p, q)=16 p(1-p-q)(2 ; 7)_{8} / 3 \\
& w_{9}(p, q)=16 p q(4 ; 10)_{9} / 3 \\
& w_{10}(p, q)=16 p q(4 ; 9)_{10} / 3 \\
& w_{11}(p, q)=16 q(1-p-q)(6 ; 12)_{11 / 3}
\end{aligned}
$$




$$
w_{12}(p, q)=16 q(1-p-q)(6 ; 11)_{12} / 3
$$

y las restantes seis funciones de base están dadas por

$$
w_{i}(s, t, p, q)=f_{i}(s, t)-\sum_{j=1}^{12} f_{i}\left(s_{j}, t_{j}\right) w_{j}(p, q)
$$

para $i=1,2, \ldots, 6$.

Si las funciones $\left\{f_{i}(s, t)\right\}, 1=1,2, \ldots, 6$ generan polinomios de segundo grado, las funciones definidas por (2) y (3) generan también poli nomios de segundo grado. Se necesita probar que en realidad las funciones definidas por (3) son cuadráticas en el contorno del elemento del pla no s-t. Probaremos ésto para la función $w_{9}(p, q)$. Consideramos la forma lineal $(3 ; 5)$. Pensada esta forma lineal como cuadrática en el plano p-q, y puesto que $p=0, q=1$ y $p=1, q=0$ pertenecen a la línea, escribimos

$$
(3 ; 5) \equiv(1-p)(1-q) \bmod (1-p-q)
$$

entonces

$$
(3 ; 5) \equiv(1-p-q+p q) \bmod (1-p-q),
$$

lo que significa que

$$
(3 ; 5) \equiv p q \bmod (1-p-q)
$$

y puesto que $p+q-1$ da el arco de parábola $(3 ; 9 ; 4 ; 10 ; 5)$ tenemos

$$
\mathrm{pq} \equiv(3 ; 5) \text { sobre el arco }(3 ; 9 ; 4 ; 10 ; 5)
$$




$$
W_{9}(p, q) \equiv \text { cuadrática en el arco de parábola }(3 ; 9 ; 4 ; 10 ; 5) \text {. }
$$

Notar que cuando la parábola se degenera a una línea recta, las formas lineales $(2 ; 7),(2 ; 8),(7 ; 8)$ coinciden. Lo mismo ocurre con $(9 ; 4,(9 ; 10),(4 ; 10) \quad y$ con $(11 ; 6),(11 ; 12),(6 ; 12)$. Estas degeneraciones producen singularidades en las funciones de base definidas en (3) puesto que, por ejemplo, no existe una forma lineal que sea cero en los nodos 2 y 8 sin ser cero en los nodos 1,7 y 3 ; así la función $w_{7}(p, q)$ no está definida en el límite cuando el arco de parábola $(1 ; 7 ; 2 ; 8 ; 3)$ se dege nera a una línea recta. Mc Leod [4] da una completa discusión sobre este tópico y se refiere a estas bases como "inestables en el límite".

\section{UNA BASE DE SEGUNDO ORDEN ESTABLE EN EL LIMTTE USANDO EL METODO HOT.}

La mayor parte de los problemas en el plano que involucran contornos curvos pueden ser resueltos usando elementos triangulares con dos lados rectos y un lado curvo, junto con triángulos de tres lados rectos. Concentraremos nuestra atención en el primer tipo de estos elementos y construiremos una base de segundo orden con límite estable usando la trans formación de alto orden descrita anteriormente.

Para ésto, consideramos el triángulo original con un lado curvo localizado en el plano $x-y$ con vértices $v_{1}\left(x_{1}, y_{1}\right), v_{2}\left(x_{2}, y_{2}\right) y v_{3}\left(x_{3}, y_{3}\right)$, donde el lado curvo es $V_{2} V_{3}$. Usando la transformación lineal

$$
\begin{aligned}
& x=\left(x_{2}-x_{1}\right) s+\left(x_{3}-x_{1}\right) t+x_{1} \\
& y=\left(y_{2}-y_{1}\right) s+\left(y_{3}-y_{1}\right) t+y_{1}
\end{aligned}
$$

el elemento original con un lado curvo es mapeado en el triángulo curvo $P_{1} P_{2} P_{3}$ en el plano $s-t$, donde los vértices son $P_{1}\left(s_{1}, t_{1}\right)=(0,0)$, $P_{2}\left(s_{2}, t_{2}\right)=(1,0), \quad P_{3}\left(s_{3}, t_{3}\right)=(0,1)$ y el lado curvo es $P_{2} P_{3}$ como se pue 
de ver en la figura 2 .

Un cuarto nodo $\left(\mathrm{x}_{4}, \mathrm{y}_{4}\right)$ es elegido en el lado curvo $\mathrm{v}_{2} \mathrm{v}_{3} \quad \mathrm{y}$ la exactitud con la cual el resultante arco parabólico, debido a la transfor mación isoparamétrica usada, aproxima el lado curvo original depende de la elección de la posición de $\left(x_{4}, y_{4}\right)$. Mc Leod y Mitchell en [5] discuten esta elección.

$$
\begin{aligned}
& \text { El jacobiano de la transformación (4) es } \\
& \qquad D=\left(x_{2}-x_{1}\right)\left(y_{3}-y_{1}\right)-\left(x_{3}-x_{1}\right)\left(y_{2}-y_{1}\right)
\end{aligned}
$$

el cual no es cero a menos que los vértices $V_{1}, V_{2} y V_{3}$ sean colineales. Usando la transformación (4), el nodo $\left(s_{4}, t_{4}\right)$ es

$$
\begin{aligned}
& s_{4}=\left[\left(y_{3}-y_{1}\right)\left(x_{4}-x_{1}\right)-\left(x_{3}-x_{1}\right)\left(y_{4}-y_{1}\right)\right] / D \\
& t_{4}=\left[\left(x_{2}-x_{1}\right)\left(y_{4}-y_{1}\right)-\left(y_{2}-y_{1}\right)\left(x_{4}-x_{1}\right)\right] / D
\end{aligned}
$$

El elemento en el plano s-t es ahora transformado no linealmente en el triángulo estandar en el plano p-q vía la transformación isopara métrica.

$$
\begin{aligned}
& s=p(1+a q) \\
& t=p(1+b p),
\end{aligned}
$$

donde a y b son determinados por la condición de que las coordenadas del punto $\left(p_{4}, q_{4}\right)$ sean $\left(\frac{1}{2}, \frac{1}{2}\right)$, es decir:

$$
\begin{aligned}
& a=2\left(2 s_{4}-1\right) \\
& b=2\left(2 t_{4}-1\right)
\end{aligned}
$$


Los nodos $\mathrm{P}_{5} \mathrm{Y} \mathrm{P}_{6}$ se eligen como $(1 / 2,0)$ y $(0,1 / 2)$ respectivamente, y por la transformación (7) los correspondientes nodos en el plano $p-q$ son $\left(p_{i}, q_{i}\right)=\left(s_{i}, t_{i}\right), i=1,2,3,5,6$ como se puede ver en la figura 2.

La transformación (7) Ileva el lado 1-p-q = 0 sobre el arco de parábola.

$$
\begin{aligned}
& \left(b s-a t+c_{1}\right)^{2}+c_{2}(a s+b t)-c_{3}=0 \\
& c_{1}=(a-b)\left(a^{2}+b^{2}-a-b\right) /\left[2\left(a^{2}+b^{2}\right)\right] \\
& c_{2}=(a+b)^{2} /\left(a^{2}+b^{2}\right) \\
& c_{3}=c_{1}^{2}+a+b+a b
\end{aligned}
$$

y está bien definida mientras el jacobiano

$$
J=1+a q+b p
$$

no sea cero.

Previo a la definición de la base de segundo orden, necesitamos un conjunto de polinomios de segundo grado en el plano s-t que interpolen exactamente polinomios de segundo grado sobre el elemento en consideración. Este conjunto de polinomios $\left\{F_{i}\right\}, i=1,2, \ldots, 6$ debe satisfacer

$$
F_{i}\left(s_{j}, t_{j}\right)=\delta_{i j} ; i, j=1,2, \ldots, 6 ;
$$

una definición adecuada es:

$$
F_{1}(s, t)=1+2\left(s^{2}+t^{2}\right)-3(s+t)-s t\left[1+\left(s_{4}^{2}+t_{4}^{2}\right)-3\left(s_{4}+t_{4}\right)\right] / s_{4} t_{4}
$$




$$
\begin{aligned}
& F_{2}(s, t)=s(2 s-1)-s t\left(2 s_{4}-1\right) / t_{4} \\
& F_{3}(s, t)=t(2 t-1)-s t\left(2 t_{4}-1\right) / s_{4} \\
& F_{4}(s, t)=s t / s_{4} t_{4} \\
& F_{5}(s, t)=4 s(1-s-t)-4 s t\left(1-s_{4}-t_{4}\right) / t_{4} \\
& F_{6}(s, t)=4 t(1-s-t)-4 s t\left(1-s_{4}-t_{4}\right) / s_{4} .
\end{aligned}
$$

Debido a que los nodos $1,2,3,4,5,6$ no pertenecen todos a una có nica, las funciones $\left\{\mathrm{F}_{i}\right\}$ son linealmente independientes $y$ generan polinonios de grado 2 en el plano s-t. También, los polinomios $\left\{F_{i}\right\}$ así defini dos generan polinomios de grado dos en el plano $x-y$. Podemos escribir las ecuaciones.

$$
\begin{aligned}
& \sum_{i=1}^{6} F_{i}(s, t)=1, \quad \sum_{i=1}^{6} s_{i} F_{i}(s, t)=s, \quad \sum_{i=1}^{6} t_{i} F_{i}(s, t)=t, \\
& \sum_{i=1}^{6} s_{i}^{2} F_{i}(s, t)=s^{2}, \quad \sum_{i=1}^{6} s_{i} t_{i} F_{i}(s, t)=s t, \sum_{i=1}^{6} t_{i}^{2} F_{i}(s, t)=t^{2}
\end{aligned}
$$

Pero para asegurar conformidad, necesita unos dos nodos extras sobre el arco parabólico. Estos nodos son elegidos como las imágenes de los nodos $\left(\frac{3}{4}, \frac{1}{4}\right)$ y $\left(\frac{1}{4}, \frac{3}{3}\right)$ bajo la transformación (7). Las coordenadas de estos dos nodos extras son

$$
\begin{aligned}
& \left(s_{7}, t_{7}\right)=(3(a+4) / 16,(3 b+4) / 16) \\
& \left(s_{8}, t_{8}\right)=((3 a+4) / 16,3(b+4) / 16) .
\end{aligned}
$$


La función de base $w_{7}$ asociada al nodo 7 debe ser idénticamente cero sobre los lados rectos, ser cero en los otros nodos del lado curvo $y$ debe tomar el valor 1 en el nodo 7. También debe reducirse a un polinomio de segundo grado sobre el lado curvo. Sea $L_{i j}$ la formalineal con valor cero en el nodo $i$, con valor uno en el nodo $j y$ con pendiente $\frac{b}{a}$, es decir, paralela al eje de la parábola (8).
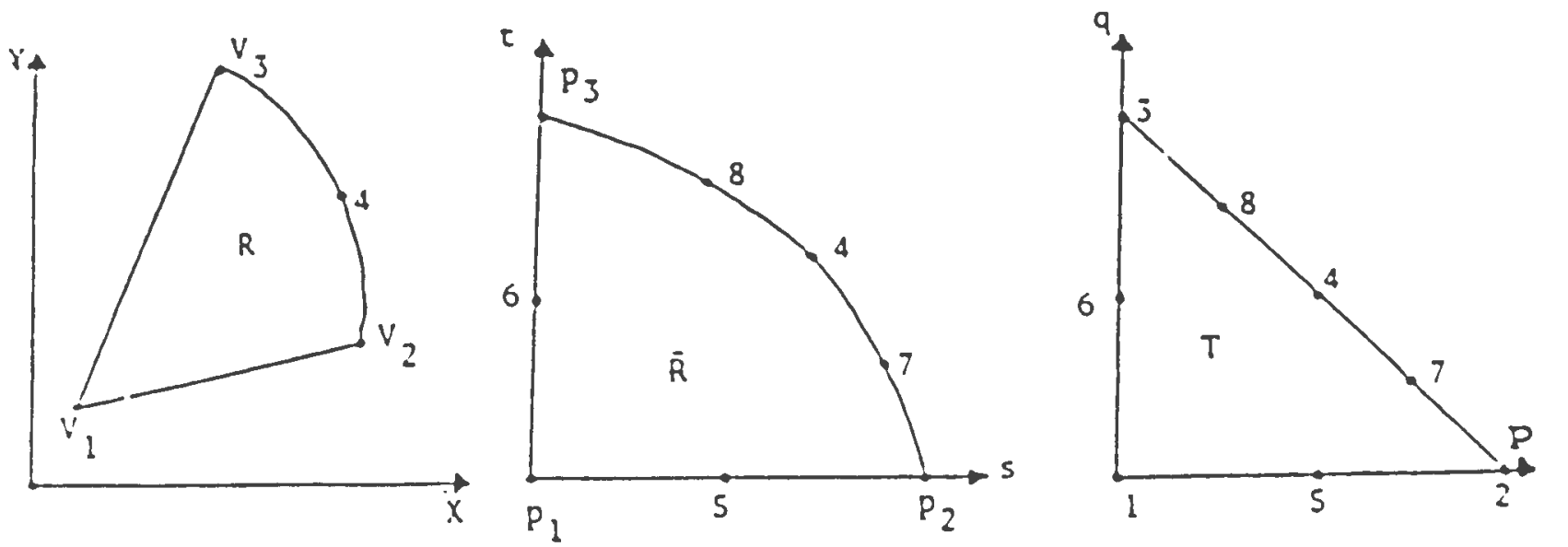

Figura 2: Configuración para una base de segundo orden usando el método HOT.

Debido a las relaciones entre $s, t \quad y \quad p, q$ dadas por las ecuacio nes (7), $I_{i j}$ puede considerarse como polinomio en $p \quad y \quad q$. Las funciones de base asociadas a los nodos 7 y 8 son

$$
\begin{aligned}
& W_{7}(p, q)=p q I_{47} I_{87} / p_{7} q_{7} \\
& W_{8}(p, q)=p q I_{48} I_{78} / p_{8} q_{8}
\end{aligned}
$$

Tenemos que probar que las funciones de base dadas por (II) son en realidad cuadráticas en el lado curvo del elemento en el plano s-t. En efecto, por las ecuaciones (7) sabemos que 


$$
\begin{aligned}
& s=p+a p q \\
& t=q+b p q, \quad \text { entonces } \\
& s+t=p+q+p q(a+b), \text { por tanto } \\
& p q \equiv(s+t-1) \bmod (p+q-1)
\end{aligned}
$$

y ésto significa que

$$
p q \equiv(s+t-1) \bmod (a r c o \text { de parábola). }
$$

También, para la función de base $w_{7}$ podemos escribir

$$
\mathrm{L}_{47} \mathrm{~L}_{87}=\mathrm{k}\left(\mathrm{bs}-\mathrm{at}-\mathrm{bs} \mathrm{s}_{4}+\mathrm{at} \mathrm{t}_{4}\right)\left(\mathrm{bs}-\mathrm{at}-\mathrm{bs} \mathrm{s}_{8}+\mathrm{at} \mathrm{t}_{8}\right)
$$

donde $\mathrm{k}$ es la constante de normalización. Entonces

$$
L_{47} L_{87}=k(b s-a t)^{2}+\operatorname{lineal}_{1}(s, t) .
$$

Pero sobre el arco de parábola tenemos que

$$
(\mathrm{bs}-\mathrm{at})^{2}=\operatorname{lineal}_{2}(s, t)
$$

Y entonces

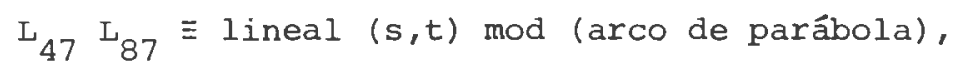

así entonces

$$
w_{7}(p, q) \equiv \text { cuadrática mod (arco de parábola). }
$$

De la misma forma podemos probar que la función de base $w_{8}(p, q)$ es cuadrá 
tica sobre el lado curvo.

Las funciones de base $W_{7} \quad y \quad W_{8}$ están bien definidas mientras la pendiente $\frac{b}{a}$ no sea $-1, y$ cuando la curva se degenera a una línea rec ta 1-s-t, ellas no presentan singularidades. Si éste es el caso, la trans formación (7) se asume ser la transformación idéntica con $a=b=0$.

Las funciones de base $W_{7} \quad y \quad W_{8}$ pueden escribirse en la forma

$$
\begin{aligned}
& \mathrm{w}_{7}(\mathrm{p}, \mathrm{q})=128 \mathrm{pq} \mathrm{w}_{1} \mathrm{w}_{2} / 3 \\
& \mathrm{w}_{8}(\mathrm{p}, \mathrm{q})=128 \mathrm{pq} \mathrm{w}_{3} \mathrm{w}_{2} / 3
\end{aligned}
$$

donde

$$
w_{j}=b^{*} p-a^{*} q+a^{*}-\frac{j}{4} ; j=1,2,3
$$

$y$ donde

$$
a^{*}=a\left|(a+b), b^{*}=b\right|(a+b)
$$

La base de segundo orden conforme y estable en el límite está dada por

$$
\begin{aligned}
w_{7}(p, q)= & 128 p q w_{1} w_{2} / 3 \\
w_{8}(p, q)= & 128 p q w_{3} w_{2} / 3 \\
w_{i}(p, q)= & F_{i}(s, t)-F_{i}\left(s_{7}, t_{7}\right) w_{7}(p, q)- \\
& F_{i}\left(s_{8}, t_{8}\right) w_{8}(p, q)
\end{aligned}
$$

para $i=1,2,3,4,5,6$. 
BIBLIOGRAFIA.

[1] GALLAGHER, R.H. Finite Elements Analysis. Prentice Hall, 1975.

[2] MC LEOD, R.J.Y. Node Requirements for the High orden Approximation over Curved Finite Elements. J. Inst. Maths. Appl. 17(1976).

[3] MC LEOD, R.J.Y. High order Transformation Methods for Curved Finite Elements. J. Inst. Maths. Appl. 21 (1978).

[4] MC LEOD, R.J.Y. On the Stability of Two-Dimensional Interpolation and High Order Basis for Curved Finite Elements. Comp. and Maths. with Appl. 5 (1979) .

[5] MC LEOD, R.J.Y., MITCHELL, A.R. The Use of Parabolic Arcs in Maching Curved Boundaries in the Finite Elements Method. J. Inst. Maths. Appl. 16 (1975).

[6] MITCHELI, A.R., WAIT, R. The Finite Element Method in Partial Diffe rential Equations. Wiley and Sons, 1977.

[7] ROJO, H.J. Basis Functions in Curved Finite Elements. Doctoral Dissertation. New Mexico State University, 1985.

[8] ROJO, H.J. Cantidad de Nodos Requeridos para Funciones de Base en Dos Dimensiones. Revista Proyecciones, Universidad del Norte, 1985.

[9] WACHPRESS, E.L. A Rational Finite Element Basis. Academic Press, 1975. 